09

\title{
Создание локализованных ансамблей NV-центров в CVD-алмазе с помощью облучения электронным пучком
}

\author{
(C) С.А. Богданов ${ }^{1}$, А.М. Горбачев ${ }^{1}$, Д.Б. Радищев ${ }^{1}$, А.Л. Вихарев ${ }^{1}$, М.А. Лобаев ${ }^{1}$, С.А. Гусев ${ }^{1}$, \\ Д.А. Татарский ${ }^{1}$, С.В. Большедворский ${ }^{2}$, А.В. Акимов ${ }^{2,3}$, В.В. Чернов ${ }^{1}$ \\ ${ }^{1}$ Федеральный исследовательский центр Институт прикладной фризики РАН, Нижний Новгород, Россия \\ ${ }^{2}$ Физический институт им. П.Н. Лебедева РАН, Москва, Россия \\ ${ }^{3}$ Texas A \& M University, College Station, USA \\ E-mail: bogser@appl.sci-nnov.ru
}

Поступило в Редакцию 11 декабря 2018г.

В окончательной редакции 18 декабря 2018г.

Принято к публикации 20 декабря 2018г.

\begin{abstract}
Продемонстрировано создание локализованных ансамблей $\mathrm{N} V$-центров, образованных в процессе облучения CVD-алмаза с легированным азотом дельта-слоем электронным пучком с энергией $200 \mathrm{keV}$ с последующим отжигом. Приведены результаты оптических измерений активированных областей при различных дозах облучения. Обсуждаются вопросы образования дефектов решетки алмаза в процессе воздействия электронного пучка.
\end{abstract}

DOI: 10.21883/PJTF.2019.06.47498.17634

Уникальные свойства $\mathrm{N} V$-центров в алмазе, такие как большие времена спиновой когерентности при комнатной температуре $\left(T_{2}\right)$, спин-зависимая флуоресценция (бесфононная линия $637 \mathrm{~nm}$ для отрицательно заряженных $\mathrm{N} V$-центров), возможность инициализации и считывания состояния электронного спина $\mathrm{N} V$-центра оптическими методами, а также управления спиновым состоянием с помощью микроволн $(2.87 \mathrm{GHz})$, создают основу для практического использования $\mathrm{N} V$-центров в ряде активно развивающихся современных направлений [1]. В настоящее время получение одиночных $\mathrm{N} V$ центров, а также массивов $\mathrm{N} V$-центров, локализованных в пространстве, с высокой точностью является одной из актуальных задач, решение которой позволит создать твердотельную среду, необходимую для задач квантовой обработки информации, квантовой коммуникации, a также для создания сверхчувствительного сенсора магнитных и электрических полей [2].

$\mathrm{N} V$-центры встречаются как в натуральном алмазе, так и в искусственном алмазе, выращенном методами НРНТ (high pressure high temperature) и CVD (chemical vapor deposition). Однако в случае натурального и НРНТ-алмаза расположение $\mathrm{N} V$-центров в образце имеет случайный характер. Только CVD-метод позволяет контролируемым образом создавать наноструктурированные ансамбли $\mathrm{N} V$-центров в процессе роста. Также активно исследуется альтернативная возможность контролируемого создания $\mathrm{N} V$-центров с помощью метода ионной имплантации $[3,4]$. Однако, несмотря на удобство создания одиночных $\mathrm{N} V$-центров, метод ионной имплантации имеет ряд ограничений, таких как разброс по глубине залегания, меньшие времена спиновой когерентности из-за локальной деформации решетки.

Дельта-легирование азотом может быть использовано для получения $\mathrm{N} V$-центров, локализованных в тонком слое нанометровой толщины, непосредственно в процессе CVD-роста. В отличие от ионной имплантации при этом не образуется дефектов решетки, ухудшающих спиновые свойства $\mathrm{N} V$-центров. Кроме того, глубина залегания конкретного исследуемого $\mathrm{N} V$-центра, созданного методом дельта-легирования, может быть определена с точностью до нескольких нанометров. Для того чтобы дельта-легирование позволяло получать ансамбли с высокой концентрацией $\mathrm{N} V$-центров, расположенных на заданной глубине, необходимо решить ряд научных задач. Прежде всего необходимо научиться создавать чрезвычайно тонкие $(2-3 \mathrm{~nm})$ легированные азотом слои с резкими границами в процессе роста CVD-алмаза и контролировать скорость роста с высокой точностью для получения повторяемых результатов, не изменяющихся от образца к образцу. Дельта-слои с $\mathrm{N} V$ центрами в CVD-алмазе уже были созданы некоторыми научными группами [5-7]. В ранее опубликованной работе нами было продемонстрировано создание дельтаслоев с высокой концентрацией азота $\left(\sim 10^{19} \mathrm{~cm}^{-3}\right)$ толщиной $3 \mathrm{~nm}$ [7]. Другой актуальной задачей является исследование методов повышения концентрации $\mathrm{N} V$ центров в уже выращенных CVD-слоях. Активно исследуются методы облучения электронным пучком [8,9], а также пучком ионов [10] для создания вакансий в алмазе. Далее образец подвергается отжигу при температуре $>800^{\circ} \mathrm{C}$ для образования $\mathrm{N} V$-центров в результате диффузии вакансий. В большинстве работ на эту тему изучалось облучение электронным пучком с высокими энергиями $(2-3 \mathrm{MeV})$ [8]. При таком подходе облучается весь образец. В настоящей работе проведено исследование возможности создания локализованных ансамблей $\mathrm{N} V$-центров с помощью облучения электронным пучком просвечивающего электронного микроскопа с энергией электронов $200 \mathrm{keV}$. За счет фокусировки, до- 


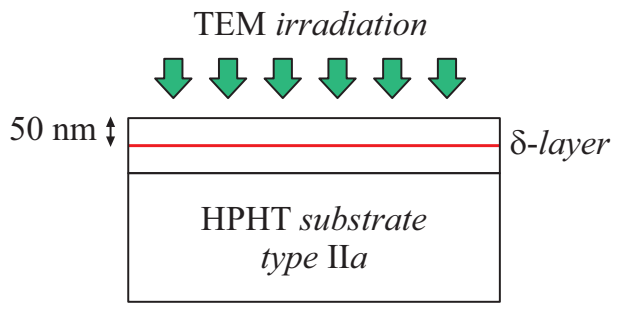

Рис. 1. Схематическое изображение облучаемого образца.

стижимой просвечивающим электронным микроскопом при облучении, возможно получение более высоких доз облучения (выше $10^{20} \mathrm{e} / \mathrm{cm}^{2}$ ) и, следовательно, большей эффективности конверсии. При таком подходе облученным оказывается не весь образец, а лишь небольшие выделенные области на нем, что позволяет создавать локализованные ансамбли с повышенной концентрацией $\mathrm{N} V$-центров.

Для проведения экспериментов были использованы подложки НРНТ-алмаза типа ІІа ориентации (100) (концентрация азота $\left.<10^{15} \mathrm{~cm}^{-3}\right)$. Использование высококачественных подложек типа IІа с крайне низким содержанием азота было необходимо для наших исследований, поскольку флуоресценция $\mathrm{N} V$-центров из подложки может затруднять изучение образования $\mathrm{N} V$-центров в CVD-слое. Исследование подложек с помощью сканирующей конфокальной микроскопии не выявило в них $\mathrm{N} V$-центров. Перед ростом подложки подвергались предварительной обработке для получения гладких и бездефектных поверхностей, которая включала в себя шлифовку и последующую обработку в плазме индукционного разряда для удаления дефектов шлифовки.

Рост дельта-слоев, легированных азотом, проводился с помощью CVD-реактора, разработанного в ИПФ РАН, в котором быстрое переключение газов и ламинарный поток газов позволяют добиваться быстрой (за несколько секунд) смены газовой смеси в реакторе, что делает возможным рост легированных слоев с резкими границами, в том числе дельта-слоев толщиной $1-2 \mathrm{~nm}$ [11]. На НРНТ-подложке NV5 нами был выращен эпитаксиальный слой с легированным азотом дельта-слоем на глубине $50 \mathrm{~nm}$ от его поверхности. Концентрация азота в дельта-слое (по измерениям методом вторичной ионной масс-спектрометрии) составляла $1.3 \cdot 10^{19} \mathrm{~cm}^{-3}$, его толщина примерно $3 \mathrm{~nm}$. С помощью просвечивающего электронного микроскопа были облучены электронами с энергией $200 \mathrm{keV}$ выделенные области образца (рис. 1). Размер облученных областей составлял $10 \mu \mathrm{m}$, расстояние между областями $300 \mu \mathrm{m}$. Доза облучения для каждой из областей была различна и изменялась в пределах $10^{19}-2 \cdot 10^{22} \mathrm{e} / \mathrm{cm}^{2}$. После облучения электронным пучком образец отжигался в вакууме при температуре $800^{\circ} \mathrm{C}$ в течение часа для формирования $\mathrm{N} V$-центров в дельта-слое вследствие диффузии вакансий. Оптические измерения облученных областей проводились с помощью конфокального микроскопа [12]. Спектр флуоресценции активированных областей исследовался с помощью спектрометра Horiba Jobin Yvon FHR-1000 c CCD-камерой Synapse CCD-2048 × 512 при комнатной температуре.

Изображение облученных областей, полученное с помощью конфокальной микроскопии, представлено на рис. 2. Результаты оптических измерений активированных областей приведены на рис. 3. Важно отметить, что при высоких дозах облучения бо́льшая часть принимаемого сигнала происходила не от $\mathrm{N} V$-центров, а от дефектов решетки алмаза, вызванных облучением. Анализ спектров флуоресценции активированных областей показал, что этими дефектами являются вакансии $V^{0}$ в нейтрально заряженном состоянии (дефект $G R 1$, $740.9 \mathrm{~nm}$ ) (рис. 3,a). Отметим, что на изображении облученных областей при умеренной дозе (рис. 2,a) видны яркие точки, соответствующие флуоресценции $\mathrm{N} V$-центров, на фоне однородной засветки, отвечающей
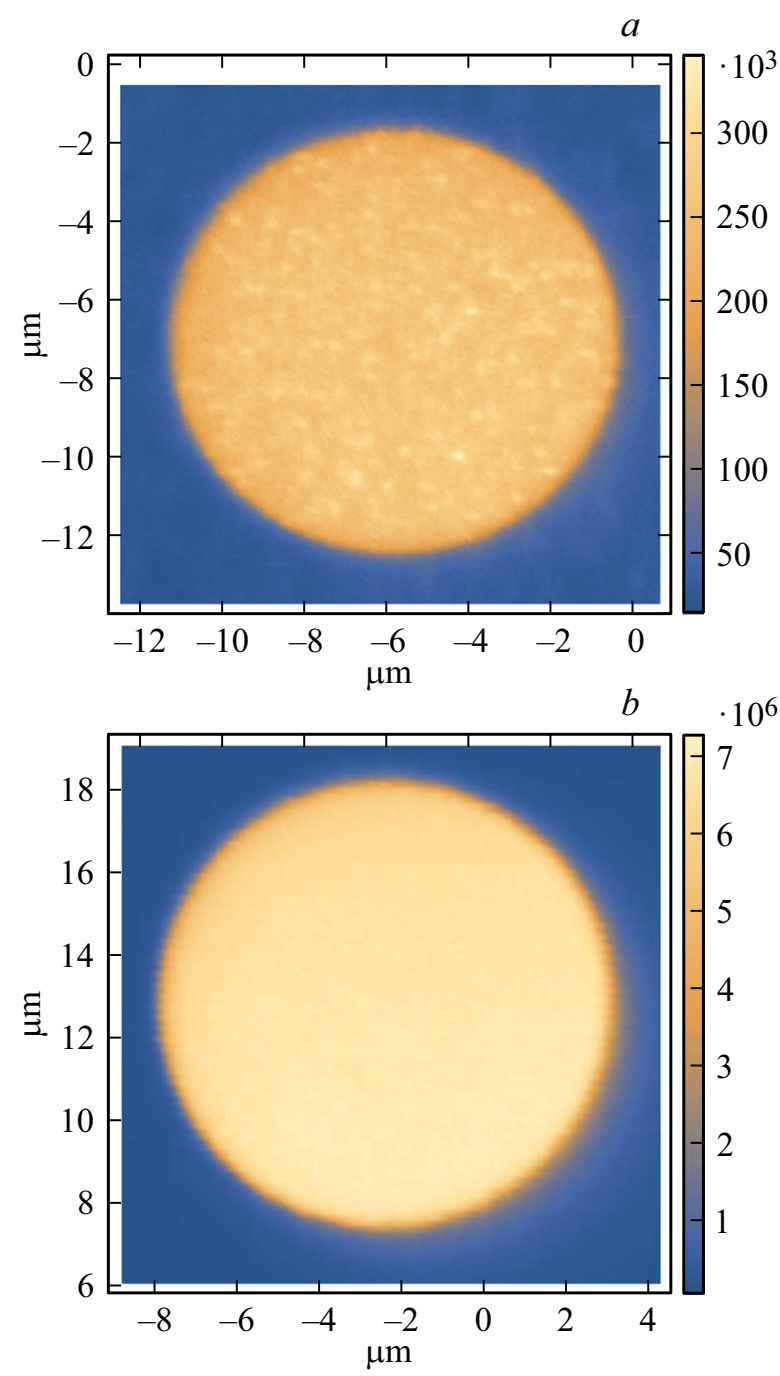

Рис. 2. Изображение облученных областей, полученное с помощью конфокальной микроскопии. Доза облучения $2 \cdot 10^{20}$ (a) и $2 \cdot 10^{22} \mathrm{e} / \mathrm{cm}^{2}(b)$. 

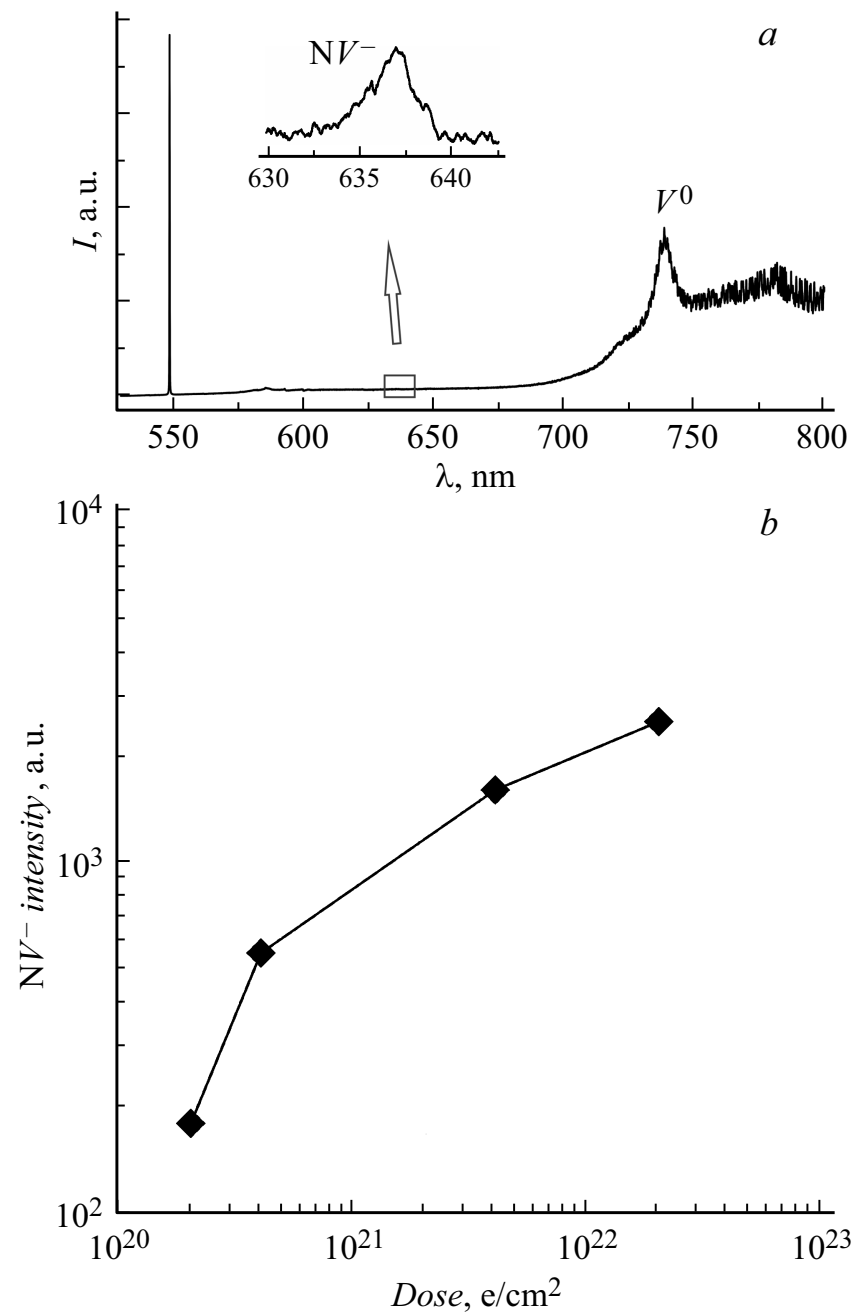

Рис. 3. $a-$ спектр флуоресценции области, облученной дозой $4 \cdot 10^{21} \mathrm{e} / \mathrm{cm}^{2} ; b-$ зависимость интенсивности флуоресценции $\mathrm{N} V^{-}$-центров от дозы облучения.

флуоресценции вакансий $V^{0}$. При наивысшей дозе облучения наблюдается практически однородный фон флуоресценции вакансий $V^{0}$ (рис. $2, b$ ). Спектры флуоресценции отчетливо демонстрируют увеличение концентрации $\mathrm{N} V$-центров в активированных областях (рис. $3, b$ ). При максимальной дозе облучения сигнал флуоресценции $\mathrm{N} V^{-}$-центров в 20 раз превосходит слабый сигнал от необлученных областей. Ранее нами было показано, что доля $\mathrm{N} V$-центров по отношению к азоту в алмазе составляет $\sim 0.2 \%$ при выбранном нами режиме роста [7]. Используя эту оценку, можно получить значение эффективности конверсии азота в $\mathrm{N} V$-центры. Эффективность конверсии растет с увеличением дозы облучения и достигает значений $\sim 4 \%$.

Отметим, что по сравнению с ранее опубликованными работами были использованы более высокие дозы облучения, а концентрация азота в дельта-слое существенно превосходила значения, достигнутые в других работах. Бо́льшая часть исследований по активации $\mathrm{N} V$-центров электронным пучком проводилась с однородно легированными образцами. После облучения в спектре флуоресценции отчетливо виден сигнал от вакансий $V^{0}$ $(740.9 \mathrm{~nm})$, который постепенно уменьшается с увеличением длительности отжига. При энергии $200 \mathrm{keV}$ электроны проникают в алмаз на значительную глубину $\sim 50 \mu \mathrm{m} \mathrm{[13].} \mathrm{Также} \mathrm{в} \mathrm{работе} \mathrm{[13]} \mathrm{показано,} \mathrm{что} \mathrm{облу-}$ чение однородно легированного образца (НРНТ-алмаз типа Ib c большой примесью азота) электронами c этой энергией приводит к образованию $\mathrm{N} V$-центров, максимальная концентрация которых наблюдается на глубине $10 \mu \mathrm{m}$. Важное отличие нашего эксперимента от ранее проведенных исследований состоит в том, что весь азот в образце сосредоточен в очень узком слое, в котором могут быть образованы $\mathrm{N} V$-центры, и созданные электронным пучком вакансии распределены в объеме, на несколько порядков большем, чем $\mathrm{N} V$-центры. Сигнал, наблюдаемый от вакансий, в нашем случае значительно превосходит сигнал от $\mathrm{N} V$-центров при больших дозах облучения. Поэтому значительный интерес представляют дальнейшие исследования отжига образцов с активированными дельта-слоями.

Таким образом, в работе продемонстрировано создание локализованных ансамблей $\mathrm{N} V$-центров в алмазе с помощью выращивания дельта-слоев, легированных азотом, и последующего облучения выделенных областей электронным пучком. Показано, что облучение образца с дельта-слоем электронами с энергией $200 \mathrm{keV}$ позволяет существенно повысить концентрацию $\mathrm{N} V$-центров в выделенных областях дельта-слоя. При максимальной дозе облучения концентрация $\mathrm{N} V$-центров возросла в 20 раз, а эффективность конверсии азота в $\mathrm{N} V$-центры составила 4\%. Разработанная методика создания локализованных ансамблей $\mathrm{N} V$-центров представляет интерес для получения наноструктур с $\mathrm{N} V$-центрами, перспективными с точки зрения приложений квантовой коммуникации и квантовой информации. Возможность контролируемого создания ансамблей $\mathrm{N} V$-центров на любой заданной глубине позволяет получать $\mathrm{N} V$-центры вблизи поверхности подложки, вплоть до нескольких нанометров, что представляет интерес для создания сверхчувствительных сенсоров магнитных и электрических полей.

Исследование выполнено за счет гранта Российского научного фонда (проект № 16-19-00163). В работе использовано оборудование ЦКП ИФМ РАН „Физика и технология микро- и наноструктур“.

\section{Список литературы}

[1] Jelezko F., Wrachtrup J. // Phys. Status Solidi A. 2006. V. 203. N 13. P. 3207-3225. DOI: $10.1002 /$ pssa.200671403

[2] Quantum information processing with diamond / Eds S. Prawer, I. Aharonovich. Elsevier, 2014. 349 p. 
[3] Aharonovich I., Santori C., Fairchild B.A., Orwa J., Ganesan K., Fu K.C., Beausoleil R.G., Greentree A.D., Prawer S. // J. Appl. Phys. 2009. V. 106. N 12. P. 124904.

[4] Naydenov B., Reinhard F., Lämmle A., Richter V., Kalish R., D'Haenens-Johansson U.F.S., Newton M., Jelezko F., Wrachtrup J. // Appl. Phys. Lett. 2010. V. 97. N 24. P. 242511.

[5] Ohno K., Heremans F.J., Bassett L.C., Myers B.A., Toyli D.M., Bleszynski Jayich A.C., Palmstrom C.J., Awschalom D.D. // Appl. Phys. Lett. 2012. V. 101. N 8. P. 082413.

[6] Lee J.C., Bracher D.O., Cui S., Ohno K., McLellan C.A., Zhang X., Andrich P., Alemán B., Russell K.J., Magyar A.P., Aharonovich I., Bleszynski Jayich A., Awschalom D., Hu E.L. // Appl. Phys. Lett. 2014. V. 105. N 26. P. 261101.

[7] Lobaev M.A., Gorbachev A.M., Bogdanov S.A., Vikharev A.L., Radishev D.B., Isaev V.A., Drozdov M.N. // Phys. Status Solidi A. 2018. V. 215. N 22. P. 1800205.

[8] Zaitsev A.M., Moe K.S., Wang W. // Diamond Related Mater. 2017. V. 71. P. $38-52$.

[9] McLellan C.A., Myers B.A., Kraemer S., Ohno K., Awschalom D.D., Bleszynski Jayich A.C. // Nano Lett. 2016. V. 16. N 4. P. $2450-2454$.

[10] Ohno K., Heremans F.J., de las Casas C.F., Myers B.A., Aleman B.J., Bleszynski Jayich A.C., Awschalom D.D. // Appl. Phys. Lett. V. 105. N 5. P. 052406.

[11] Lobaev M.A., Gorbachev A.M., Bogdanov S.A., Vikharev A.L., Radishev D.B., Isaev V.A., Chernov V.V., Drozdov M.N. // Diamond Related Mater. 2017. V. 72. P. 1-6.

[12] Bolshedvorskii S.V., Vorobyov V.V., Soshenko V.V., Shershulin V.A., Javadzade J., Zeleneev A.I., Komrakova S.A., Sorokin V.N., Belobrov P.I., Smolyaninov A.N., Akimov A.V. // Opt. Mater. Express. 2017. V. 7. N 11. P. 4038-4049.

[13] Kim E., Acosta V.M., Bauch E., Budke D., Hemmer P.R. // Appl. Phys. Lett. 2012. V. 101. N 8. P. 082410. 